\title{
Pretreatment texture analysis of routine MR images and shape analysis of the diffusion tensor for prediction of volumetric response after radiosurgery for meningioma
}

\author{
Herwin Speckter, MSc, ${ }^{1,2}$ Jose Bido, MD, ${ }^{1}$ Giancarlo Hernandez, MD, ${ }^{1}$ Diones Rivera, MD,1 \\ Luis Suazo, MD, ${ }^{1}$ Santiago Valenzuela, MD, Isidro Miches, MD, ${ }^{1}$ Jairo Oviedo, MSc, ${ }^{2}$ \\ Cesar Gonzalez, MD, ${ }^{2}$ and Peter Stoeter, MD ${ }^{1,2}$ \\ ${ }^{1}$ Centro Gamma Knife Dominicano and 2Department of Radiology, CEDIMAT, Plaza de la Salud, Santo Domingo, \\ Dominican Republic
}

OBJECTIVE The goal of this study was to identify parameters from routine T1- and T2-weighted MR sequences and diffusion tensor imaging (DTI) that best predict the volumetric changes in a meningioma after treatment with Gamma Knife radiosurgery (GKRS).

METHODS In 32 patients with meningioma, routine MRI and DTI data were measured before GKRS. A total of 78 parameters derived from first-level texture analysis of the pretreatment MR images, including calculation of the mean, SD, 2.5th and 97.5th percentiles, and kurtosis and skewness of data in histograms on a voxel-wise basis, were correlated with lesion volume change after a mean follow-up period of 3 years (range 19.5-63.3 months).

RESULTS Several DTI-derived parameters correlated significantly with a meningioma volume change. The parameter that best predicted the results of GKRS was the 2.5th percentile value of the smallest eigenvalue $(\mathrm{L} 3)$ of the diffusion tensor (correlation coefficient $0.739, p \leq 0.001$ ), whereas among the non-DTI parameters, only the SD of T2-weighted images correlated significantly with a tumor volume change (correlation coefficient $0.505, p \leq 0.05$, after correction for family-wise errors using false-detection-rate correction).

CONCLUSIONS DTI-derived data had a higher correlation to shrinkage of meningioma volume after GKRS than data from T1- and T2-weighted image sequences. However, if only routine MR images are available, the SD of T2-weighted images can be used to predict control or possible progression of a meningioma after GKRS.

https://thejns.org/doi/abs/10.3171/2018.7.GKS181327

KEYWORDS texture analysis; diffusion tensor imaging; meningioma; Gamma Knife; stereotactic radiosurgery; oncology

$\mathrm{T}$ EXTURE analysis of MRI data from cerebral tumors has been shown to be an important tool for characterizing the microenvironment within these neoplasms, and after the first efforts to correlate histopathology with contrast enhancement, "radiomics" has turned out to be a promising but still-emerging concept in radiation oncology. 1,13,17 This tool applies to not only intracerebral malignancies (e.g., glioblastomas and metastase ${ }^{3,8,15}$ ) but also benign extracerebral growths (e.g., meningiomas).

In an early study from 1992, Chen et al. ${ }^{4}$ showed a significant correlation between increased vascularity or cel- lular atypia found on T2-weighted images and in histopathological findings, and Filippi et al. ${ }^{7}$ differentiated lowand high-grade meningiomas by using diffusion weighted imaging. Later, diffusion tensor imaging (DTI) enabled differentiation between histological subtypes, which was based mainly on the calculation of fractional anisotropy (FA) and the shape of the diffusion tensor. ${ }^{11,22}$ Finally, using histograms from 8 different DTI parameters and data from T1- and T2-weighted images, the best model was elaborated statistically to classify meningiomas according to their subtype and grade. ${ }^{24}$

ABBREVIATIONS $\mathrm{CC}=$ correlation coefficient; $\mathrm{CE}=$ contrast enhanced; $\mathrm{Cl}=$ longitudinal index; $\mathrm{Cp}=$ planar index; $\mathrm{Cs}=$ spherical index; $\mathrm{DTI}=$ diffusion tensor imaging; $\mathrm{FA}$ = fractional anisotropy; GKRS = Gamma Knife radiosurgery; SK = skewness of tensor shape; SRS = stereotactic radiosurgery.

SUBMITTED May 13, 2018. ACCEPTED July 19, 2018.

INCLUDE WHEN CITING DOI: 10.3171/2018.7.GKS181327. 
Carrying this concept to clinical application in radiosurgery, Speckter et al..$^{19}$ described a significant predictive value of FA and other DTI parameters, measured before treatment, for volume reduction after Gamma Knife radiosurgery (GKRS) in meningiomas. In their study, only the mean values of 6 different DTI parameters were compared to predict the success of GKRS, and T1- and T2weighted image sequences were not included. However, T1- and T2-weighted imaging is still the workhorse of brain tumor MRI; to date, DTI has rarely been used in routine imaging.

We conducted our study for 2 reasons, 1) to look for additional (and possibly better) correlations between DTI parameters and volumetric tumor response by including skewness of tensor shape (SK) and evaluating additional features of the data histograms as the percentile distribution and kurtosis and 2) to investigate whether histogram analysis of routine MRI sequences can contribute to predicting the outcome of GKRS.

\section{Methods}

This partially retrospective study was approved by the institutional review board at CEDIMAT hospital and included some pre-GKRS imaging data from the Speckter et al. ${ }^{19}$ study mentioned earlier. However, tumor volume changes in these patients were updated according to more recent follow-up examination results.

\section{Patients and Meningiomas}

Included were 32 patients between 26.8 and 80.4 years of age with an imaging-diagnosed tumor that we believed radiographically represented a benign meningioma and treated at our Gamma Knife center. MRI had been performed within 2 months before their GKRS, and followup data from at least 1 MR scan after an interval of 18 months or more (range 19.5-63.3 months) were available (mean follow-up period 3 years) (Table 1). Twenty-three meningiomas were localized at the cranial base, 2 at the tentorium, and 7 at the convexity. We were able to retrieve histology results from 6 of 7 cases in which a preceding operation was documented; 4 of the tumors were characterized as meningothelial and psammomatous meningioma and 2 as a fibroblastic subtype, all without atypical features. Tumor sizes ranged between 0.6 and $22.5 \mathrm{~cm}^{3}$ (mean $8.7 \mathrm{~cm}^{3}$ ).

\section{Gamma Knife Treatment}

GKRS was performed on a Leksell Gamma Knife unit (model 4C, Elekta). Depending on tumor size and localization, the margin dose varied from 9.7 to 18 Gy (Table 1). The treatment was planned on a Leksell GammaPlan 10.1 workstation (Elekta) to carefully avoid undue radiation to sensitive structures such as the optic system or the cochlea and to keep the coverage index as high as possible (mean 96.8\%). The margin dose was 12-18 Gy (mean 13.9 Gy) applied in a single session with the exception of 1 case, which was treated by hypofractionated GKRS (3 $\times 5$ Gy, corresponding to a single fraction equivalent dose of $9.7 \mathrm{~Gy}$, applying an $\alpha / \beta$ ratio of $3.76 \mathrm{~Gy}$, according to Vernimmen and Slabbert ${ }^{23}$ ).
TABLE 1. Patient characteristics

\begin{tabular}{lc}
\hline \multicolumn{1}{c}{ Characteristic } & Data (range) \\
\hline No. of patients & 32 \\
\hline Mean age in yrs & $57.5(26.8$ to 80.4$)$ \\
\hline Mean tumor vol in $\mathrm{cm}^{3}$ & $8.73(0.6$ to 22.5$)$ \\
\hline Mean margin dose in Gy & $13.9(9.7$ to 18.0$)$ \\
\hline Mean follow-up in mos & $35.7(19.5$ to 63.3$)$ \\
\hline Mean vol change per mo in \% & $-0.92(-2.54$ to 0.74$)$ \\
\hline
\end{tabular}

\section{MRI}

MRI was performed on a 3-T scanner (Achieva, Philips). Apart from 3D T1- and routine T2-weighted sequences before and after the injection of contrast medium, the following sequence parameters were measured in each DTI pretreatment scan: gradient directions $32 ; \mathrm{b}=0$ and $800 \mathrm{~mm}^{2} / \mathrm{second}$; measured voxel size $2 \times 2 \times 2 \mathrm{~mm} ; 60$ slices covering the whole head; SENSE factor 2; and acquisition time 4.5 minutes.

\section{Postprocessing}

Tumor volumes were outlined and measured from 3D T1-weighted contrast-enhanced images on the Leksell GammaPlan workstation.

From the DTI data, b0 images, and maps of FA, median, axial, and radial diffusivity values were calculated, as were the longitudinal, planar, and spherical indices of the tensor ellipsoids (longitudinal index [Cl], planar index $[\mathrm{CP}]$, and spherical index [Cs], respectively) and the 3 eigenvalues L1 (equivalent to axial diffusivity), L2, and L3, respectively, using the program ExploreDTI (http:// exploredti.com).$^{14}$ From the histograms of L1, L2, and L3 data, a voxel-wise SK map was calculated. SK corresponds to the third moment of the eigenvalues ${ }^{5}$ and is related to the shape of the diffusion ellipsoid.

MRIcro software (https://www.mccauslandcenter. sc.edu/crnl/) was used to outline regions of interest interactively on b0 images covering the tumor. After transference to the DTI parameter maps, the corresponding values were measured as histograms. In the same way, data were collected from T1-weighted images before and after the injection of contrast medium and from T2-weighted images and related to the means taken from 6 different regions of interest placed into the genu and splenium of the corpus callosum of the same images.

For all meningiomas, a total of 3 histograms were derived from conventional imaging, and another 10 histograms were derived from DTI data sets. From the data from all 13 histograms, the following 6 parameters were calculated: mean, SD, 2.5th and 97.5th percentile, skewness (of the histogram), and kurtosis (Figs. 1 and 2), giving a total data set that included 78 parameters. In a second step, these 78 parameters were correlated with lesion volume change in each of the 32 meningiomas after a mean follow-up period of 3 years.

Using the functionality of the statistical package SPSS 15.0 (SPSS, Inc.), we performed a partial correlation analysis and correlated the change in tumor volume after GKRS to the various DTI parameters after correcting for the follow-up period, lesion size, margin dose, and patient age, 


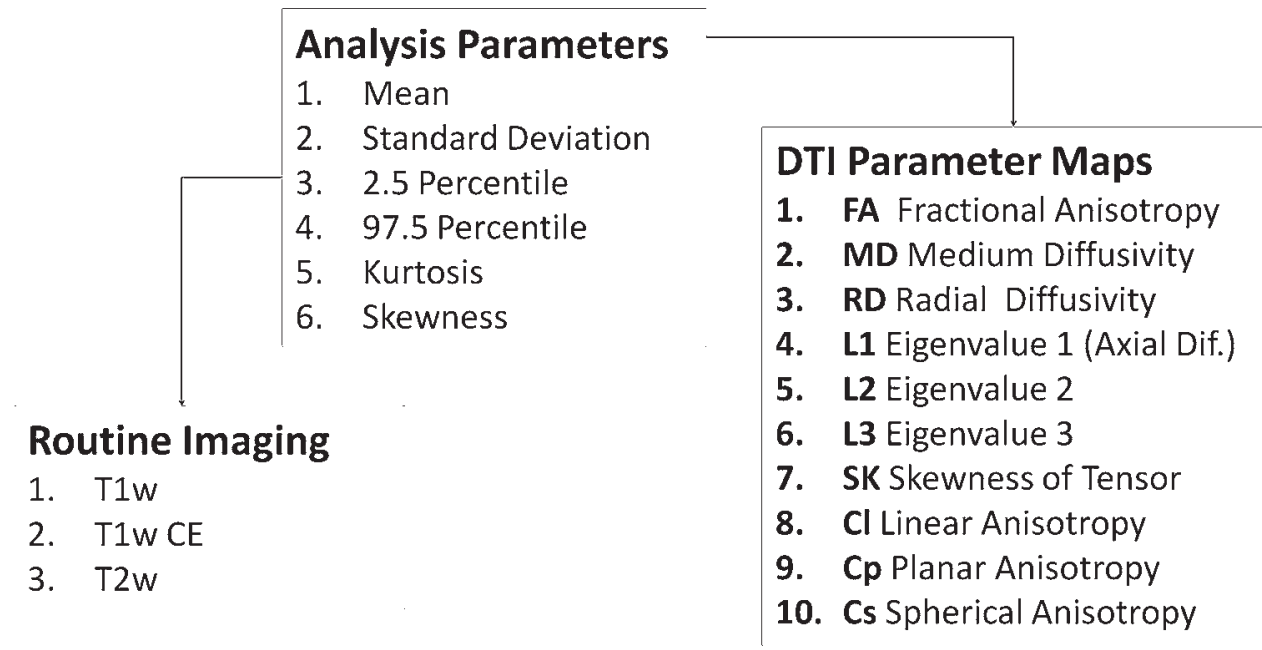

FIG. 1. Analysis parameters applied to routine imaging and DTI parameter maps in this study. $\mathrm{CE}=$ contrast enhanced; $\mathrm{T} 1 \mathrm{w}=\mathrm{T} 1$ weighted; $\mathrm{T} 2 \mathrm{w}=\mathrm{T} 2$ weighted.

entered as control variables. We also conducted a linear regression analysis with tumor volume change per month as a dependent variable and the previously mentioned parameters as independent variables to determine which parameter or combination of parameters could best explain the variance in volume change. Because 78 data sets were included, results of the significance calculation had to be corrected for family-wise error using false-detection-rate correction (www.sdmproject.com/utilities/?show=FDR). ${ }^{2}$ The level of significance was set to $95 \%$.

\section{Results}

After a follow-up period of at least 18 months (mean 3 years [35.7 months]), the mean tumor volume reduction was $29 \% \pm 25.4 \%$, which amounts to a rate of $0.92 \%$ per month (Table 1). At the last follow-up, 27 of 32 meningiomas had regressed, 4 had stayed stable within a $\pm 15 \%$ volume change range, ${ }^{9}$ and 1 meningioma had progressed (Table 2 and Fig. 3).

After we adjusted for patient age, tumor size, margin dose during GKRS, and follow-up period, we found a significant negative correlation between the reduction in tumor volume and mean FA values measured before GKRS (correlation coefficient $[\mathrm{CC}]-0.639, \mathrm{p} \leq 0.001$ ). A significant negative correlation was found between change in tumor volume and $\mathrm{Cp}$ and the calculated skewness values (CC -0.675 and $-0.680, \mathrm{p} \leq 0.001$ for both), whereas the correlation to the Cs was positive (CC $-0.646, \mathrm{p} \leq 0.001)$ (Table 3). Among the other parameters calculated from the histograms, the 2.5th and 97.5th percentiles of Cp and Cs had highly significant correlations to tumor shrinkage ( $p \leq 0.001)$, as did the 2.5th percentiles from SK and FA. The highest $\mathrm{CC}$ of the whole study (0.739) was that of the 2.5th percentile of L3, the smallest eigenvalue (Fig. 4).

Most of the DTI parameters clearly identified the only meningioma that presented progression in this study (Table 2). This meningioma progressed continuously after GKRS; the volume had increased by $45.0 \%(0.74 \%$ / month) at the latest follow-up visit, which was performed
60.4 months after GKRS. Most DTI parameters presented an extreme value for this meningioma, outside the region of values for stable meningiomas or for those that have regressed (Table 2).

From the parameters calculated from the non-DTI data, only the SD values from T2-weighted images correlated significantly with tumor change per month (CC 0.505, p $\leq 0.05$ ). The $\mathrm{SD}$ of $\mathrm{T} 2$-weighted images was lowest for the only meningioma that progressed (Table 2 and Fig. 4). Meningiomas with a poorer response to GKRS can be identified directly on T2-weighted images, because their depiction is less homogenous than that for meningiomas that respond well to GKRS (Fig. 5)

Linear correlation analysis with the inclusion of the 9 parameters with the highest CCs and the SD of T2-weighted images as independent variables and volume change per month as a dependent variable revealed an important contribution of the 2.5th percentile from FA data to explain $49.2 \%$ of the variance in tumor change, which increased to $59.2 \%$ by adding the SD of T2-weighted images.

We examined other factors for correlation with volume regression, including initial tumor size, margin dose, tumor coverage, patient age, and follow-up period. Of these parameters, patient age, initial tumor size, and follow-up period correlated negatively, whereas margin dose and tumor coverage correlated positively with posttreatment reduction in tumor size but did not reach significance (initial tumor size, $\mathrm{CC}-0.12$; margin dose, CC 0.10 ; tumor coverage, $\mathrm{CC} 0.03$; patient age, $\mathrm{CC}-0.32$; and follow-up period, CC -0.33 ).

\section{Discussion}

Conventional resection of meningiomas is frequently preferred currently over stereotactic radiosurgery (SRS). Prediction of volumetric control after SRS can provide important information for selecting an adequate treatment strategy. A predicted fast tumor-shrinkage rate can offer an additional argument in favor of SRS. Also, in cases of a predicted good response, one might reduce the 


\section{Histogram}
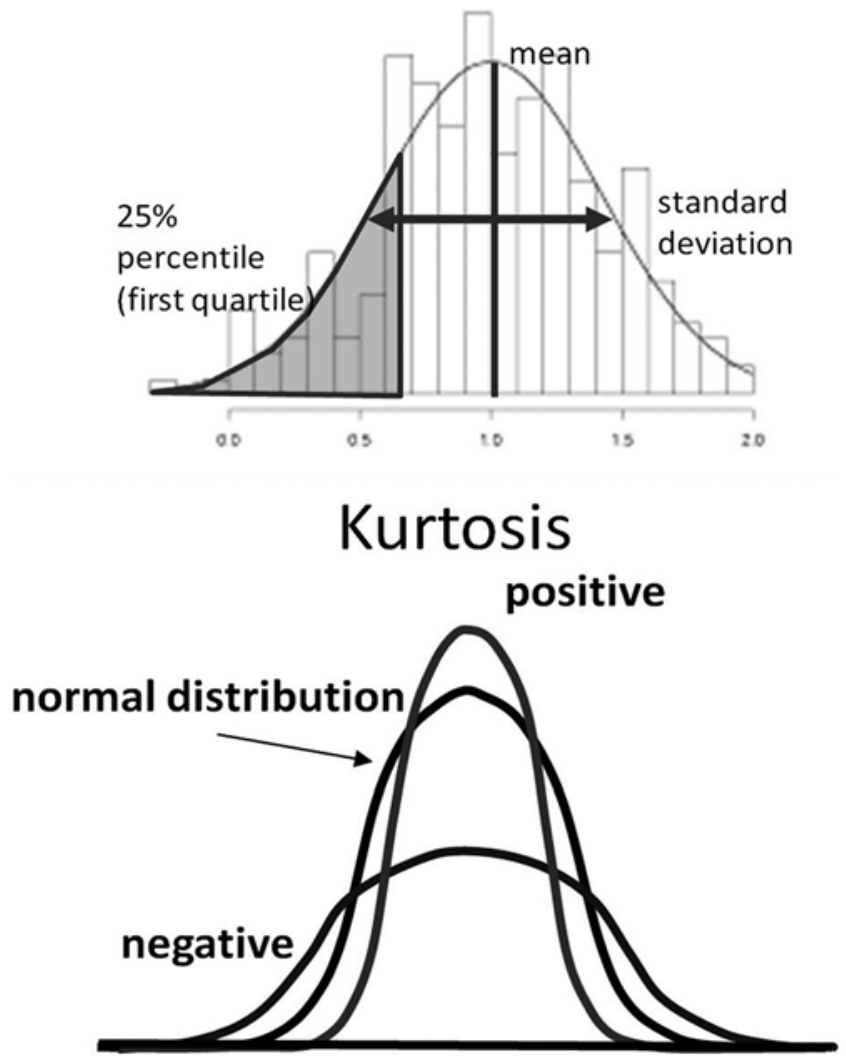

\section{Skewness}

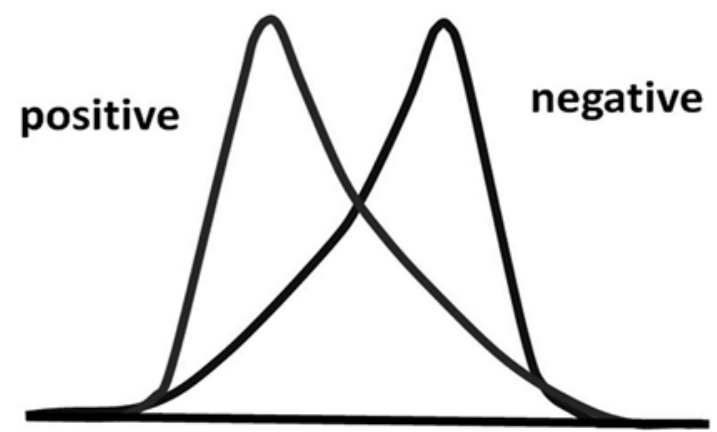

FIG. 2. Graphic representation of histogram and texture analysis parameters applied in this study.



FIG. 3. Waterfall plot of eventual volume changes. At the time of last follow-up, 27 of 32 meningiomas had regressed, 4 had stayed stable, and 1 had progressed.

radiation dose to reduce the risk of adverse radiation effects. In contrast, if tumor progression after SRS is predicted, one might consider increasing the radiation dose or ponder performing conventional resection.

Texture analysis based on MRI data revealed a highly significant correlation between DTI data, mainly FA, Cp, and L3, and the rate of tumor volume reduction per month. In this way, previous results ${ }^{19}$ were confirmed by including a larger number of patients, a longer follow-up period, and the application of a different software package for calculating DTI parameters.

Again, the meningioma with highest mean FA value was the only meningioma that progressed after GKRS. Stable controlled meningiomas, those that stayed within the $\pm 15 \%$ limit of volume variation, ${ }^{9}$ presented relatively high FA values. By including additional DTI parameters and histogram-evaluation methods (SD, percentiles, kurtosis, and skewness), tissue pattern analysis was set on a broader basis and thus identified the 2.5th percentiles of $\mathrm{FA}, \mathrm{Cp}$, and $\mathrm{L} 3$ values as the 3 parameters with the highest correlations to tumor change after GKRS. FA and Cp had been demonstrated to differentiate between fibroblastic and other subtypes, ${ }^{11,22}$ whereas SK was the best parameter for identifying meningiomas of a higher grade. ${ }^{24}$

Apart from DTI parameters, data from sequences of routine MRI examinations were included in the study in the form of T1-weighted images created before and after the injection of contrast medium and T2-weighted images. However, only 1 of these parameters, the SD of T2-weighted images, correlated significantly with tumor volume change after GKRS. In previous studies, increased

TABLE 2. Tumor characteristics and outcomes

\begin{tabular}{lcccccc}
\hline \multicolumn{1}{c}{ Outcome } & $\begin{array}{c}\text { No. of Pts } \\
(\%)\end{array}$ & $\begin{array}{c}\text { Margin Dose, Gy } \\
\text { (mean [range]) }\end{array}$ & $\begin{array}{c}\text { Initial Tumor Vol, } \mathrm{cm}^{3} \\
\text { (mean [range]) }\end{array}$ & $\begin{array}{c}\text { Reduction in Vol/mo } \\
\text { (mean \%) }\end{array}$ & $\begin{array}{c}\text { SD of T2-Weighted Data } \\
\text { (mean [range]) }\end{array}$ & $\begin{array}{c}\text { FA } \\
\text { (mean [range]) }\end{array}$ \\
\hline Tumor progression & $1(3)$ & 14.0 & 7.87 & -0.74 & 47 & 0.44 \\
\hline Stable control & $4(13)$ & $12.9(9.7-15.0)$ & $10.53(5.2-18.4)$ & 0.04 & $88(62-102)$ & $0.37(0.31-0.41)$ \\
\hline Tumor regression & $27(84)$ & $14.1(12.0-18.0)$ & $8.50(0.6-22.5)$ & 1.11 & $118(43-283)$ & $0.23(0.15-0.35)$ \\
\hline
\end{tabular}

Pts $=$ patients. 
TABLE 3. DTI parameters before GKRS and correlation to change in tumor volume

\begin{tabular}{|c|c|c|c|c|c|c|}
\hline & Mean & SD & 2.5th Percentile & 97.5th Percentile & Kurtosis & Skewness \\
\hline T1w & $0.639 \pm 0.125$ & $0.072 \pm 0.028$ & $0.595 \pm 0.125$ & $0.686 \pm 0.129$ & $2.225 \pm 2.726$ & $-0.469 \pm 0.503$ \\
\hline $\mathrm{CC}$ & -0.217 & -0.354 & -0.261 & -0.15 & -0.292 & -0.053 \\
\hline CE T1w & $1.755 \pm 0.526$ & $0.264 \pm 0.146$ & $1.611 \pm 0.512$ & $1.915 \pm 0.584$ & $2.880 \pm 2.615$ & $-0.220 \pm 0.891$ \\
\hline CC & 0.360 & 0.367 & 0.300 & 0.395 & 0.033 & -0.128 \\
\hline $\mathrm{T} 2 \mathrm{w}$ & $1.423 \pm 0.193$ & $0.176 \pm 0.088$ & $1.312 \pm 0.195$ & $1.547 \pm 0.211$ & $1.237 \pm 2.111$ & $-0.404 \pm 0.560$ \\
\hline $\mathrm{CC}$ & 0.091 & $0.505^{*}$ & -0.115 & 0.298 & 0.044 & -0.125 \\
\hline FA & $0.255 \pm 0.076$ & $0.096 \pm 0.024$ & $0.186 \pm 0.065$ & $0.312 \pm 0.092$ & $1.284 \pm 2.316$ & $0.670 \pm 0.656$ \\
\hline $\mathrm{CC}$ & $-0.639 \ddagger$ & -0.252 & $-0.686 \ddagger$ & $-0.634 \ddagger$ & 0.353 & $0.531^{*}$ \\
\hline $\mathrm{MD}$ in $10^{-6} \times \mathrm{mm}^{2} / \mathrm{sec}$ & $0.965 \pm 0.261$ & $0.192 \pm 0.099$ & $0.856 \pm 0.235$ & $1.041 \pm 0.300$ & $8.657 \pm 9.598$ & $1.656 \pm 1.415$ \\
\hline $\mathrm{CC}$ & $0.553 \dagger$ & 0.326 & $0.556 \dagger$ & $0.539^{*}$ & -0.029 & -0.129 \\
\hline $\mathrm{RD}$ in $10^{-6} \times \mathrm{mm}^{2} / \mathrm{sec}$ & $0.848 \pm 0.246$ & $0.191 \pm 0.097$ & $0.740 \pm 2.205$ & $0.929 \pm 0.290$ & $7.477 \pm 8.613$ & $1.363 \pm 1.435$ \\
\hline $\mathrm{CC}$ & $0.600 \dagger$ & 0.339 & $0.595 \dagger$ & $0.579 \dagger$ & -0.029 & -0.129 \\
\hline$A D$ in $10^{-6} \times \mathrm{mm}^{2} / \mathrm{sec}$ & $1.197 \pm 0.304$ & $0.230 \pm 0.101$ & $1.058 \pm 0.273$ & $1.296 \pm 0.343$ & $7.023 \pm 8.392$ & $1.539 \pm 1.195$ \\
\hline CC & $0.459^{*}$ & 0.276 & $0.474^{*}$ & 0.440 & 0.041 & -0.050 \\
\hline L2 in $10^{-6} \times \mathrm{mm}^{2} / \mathrm{sec}$ & $0.975 \pm 0.267$ & $0.206 \pm 0.094$ & $0.850 \pm 0.246$ & $1.067 \pm 0.308$ & $6.531 \pm 7.737$ & $1.376 \pm 1.265$ \\
\hline $\mathrm{CC}$ & $0.426^{*}$ & 0.254 & $0.439^{*}$ & $0.397^{*}$ & 0.097 & -0.056 \\
\hline $\mathrm{L} 3$ in $10^{-6} \times \mathrm{mm}^{2} / \mathrm{sec}$ & $0.734 \pm 0.259$ & $0.206 \pm 0.100$ & $0.610 \pm 0.244$ & $0.835 \pm 0.300$ & $5.421 \pm 6.416$ & $0.947 \pm 1.302$ \\
\hline $\mathrm{CC}$ & $0.729 \ddagger$ & 0.29 & $0.739 \ddagger$ & $0.689 \ddagger$ & 0.051 & -0.074 \\
\hline SK in $10^{-18} \times \mathrm{mm}^{4} / \mathrm{sec}^{2}$ & $-2.156 \pm 6.740$ & $10.511 \pm 7.665$ & $-5.803 \pm 1.010$ & $1.622 \pm 2.569$ & $31.63 \pm 49.19$ & $1.066 \pm 3.890$ \\
\hline $\mathrm{CC}$ & $0.680 \ddagger$ & -0.379 & $0.643 \ddagger$ & $0.626 \dagger$ & 0.076 & 0.241 \\
\hline $\mathrm{Cl}$ in $\%$ & $18.6 \pm 3.6$ & $9.3 \pm 1.6$ & $11.7 \pm 2.6$ & $24.5 \pm 4.6$ & $0.376 \pm 1.175$ & $0.567 \pm 0.369$ \\
\hline $\mathrm{CC}$ & -0.053 & -0.141 & -0.045 & -0.039 & -0.161 & -0.158 \\
\hline Cp in \% & $21.0 \pm 9.9$ & $11.0 \pm 3.6$ & $12.8 \pm 7.6$ & $27.9 \pm 12.8$ & $1.120 \pm 1.932$ & $0.810 \pm 0.508$ \\
\hline $\mathrm{CC}$ & $-0.675 \ddagger$ & $-0.552 \dagger$ & $-0.682 \ddagger$ & $-0.676 \ddagger$ & 0.442 & $0.601 \dagger$ \\
\hline Cs in $\%$ & $60.4 \pm 10.6$ & $12.5 \pm 2.8$ & $52.4 \pm 12.5$ & $69.2 \pm 9.6$ & $0.640 \pm 1.602$ & $-0.415 \pm 0.547$ \\
\hline $\mathrm{CC}$ & $0.646 \ddagger$ & -0.225 & $0.637 \ddagger$ & $0.682 \ddagger$ & 0.300 & $-0.505 \dagger$ \\
\hline
\end{tabular}

$\mathrm{AD}=$ axial diffusivity; $\mathrm{CE}$ = contrast enhanced; $\mathrm{MD}=$ median diffusivity; $\mathrm{RD}$ = radial diffusivity; $\mathrm{T} 1 \mathrm{w}=\mathrm{T} 1$-weighted images; $\mathrm{T} 2 \mathrm{w}=\mathrm{T} 2$-weighted images .

${ }^{*} p \leq 0.05$.

$\dagger p \leq 0.01$.

$\ddagger p \leq 0.001$

$\mathrm{T} 2$ values were reported to relate to a soft consistency of the meningioma, increased vascularity, cellular atypia, and angioblastic or melanocytic components, ${ }^{4,16,20}$ as well as cystic degeneration and ischemic necrosis. ${ }^{10}$ Because the SD was calculated in this study on a voxel-wise basis, high SD values might represent a decrease in tumor microenvironment homogeneity.

According to regression analysis, the SD of T2-weight-

\section{SD (T2) versus Volume Change / Month}



2.5 Percentile (L3) versus Volume Change / Month



FIG. 4. Strongest correlations to volume change per month. Left: Volume change per month correlates to the SD of T2-weighted images $(C C=0.505, p=0.004)$. Right: Volume change per month correlates to the 2.5 th percentile of the eigenvalue $L 3(C C=$ $0.739, p<0.001)$. 

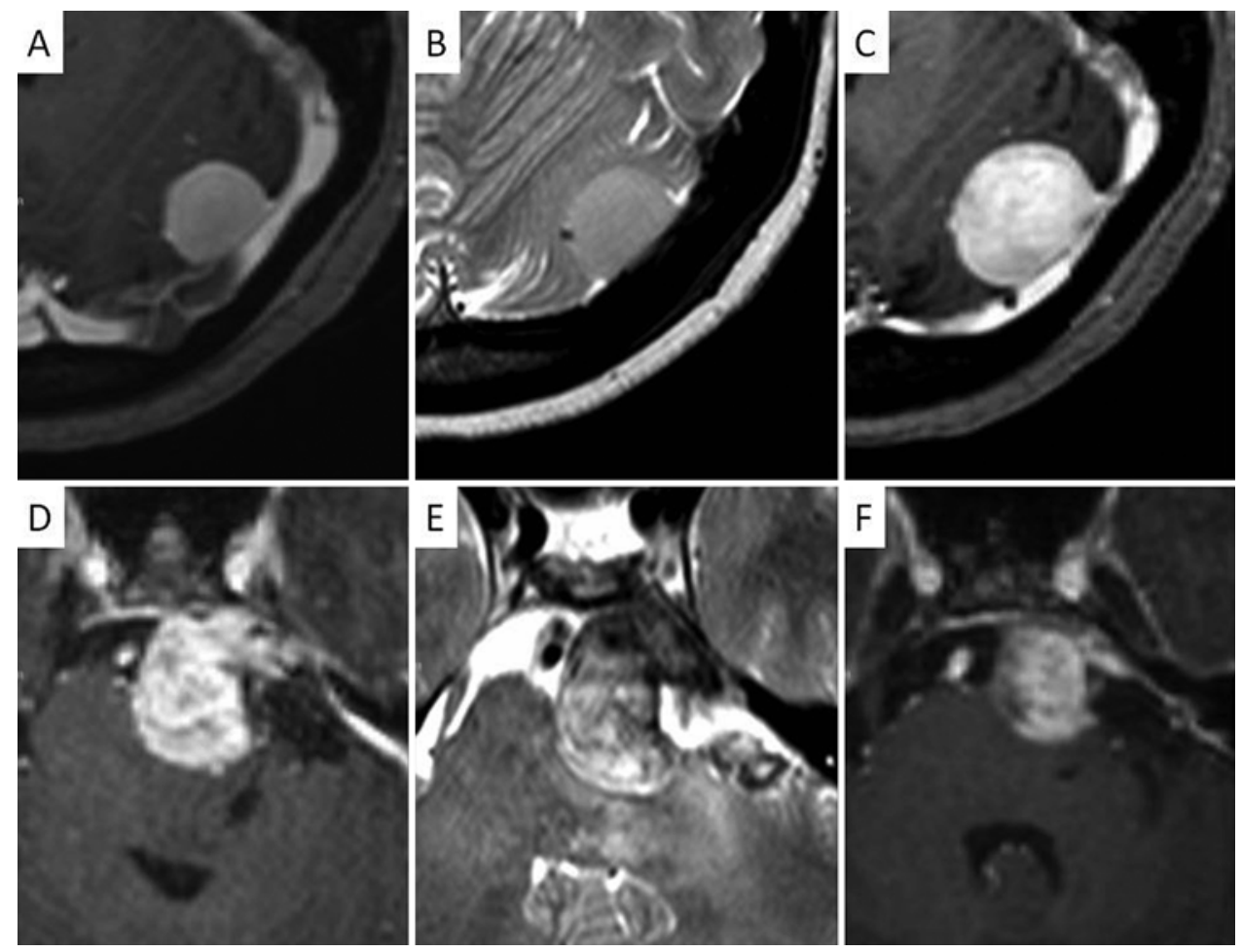

FIG. 5. Examples of conventional MR images of 2 meningiomas. A-C: Meningioma with volume progression of $0.74 \% /$ month, presenting a low SD of T2-weighted images (47). A: CE T1-weighted image before SRS. B: T2-weighted image before SRS. C: CE T1-weighted image 31 months after SRS. D-F: Meningioma with significant volume regression of $1.87 \% /$ month, presenting a high SD of T2-weighted images (283). D: CE T1-weighted image before SRS. E: T2-weighted image before SRS. F: CE T1-weighted image 60 months after SRS.

ed data from anatomical images and FA from DTI images both contribute to the variance or "explain" the variance of posttreatment change in tumor volume. A cross-correlation analysis between both parameters revealed a very low $\mathrm{CC}$ of -0.19 . Although both values are predictive for tumor control, the insignificant cross-correlation indicates that there are 2 independent mechanisms involved. Increased vascularity, cellular atypia, and angioblastic or melanocytic components, which are detected with T2-weighted images, are probably independent of fiber structure detected with DTI. The results of previous studies suggest that DTI parameters can differentiate between meningioma subtypes..$^{11,22}$ Based on this observation, one can combine these 2 independent values to improve the predictive value of imaging for tumor control with radiosurgery.

Pre-GKRS histology results were available in only 6 of our 32 cases, so we could not draw any conclusions related to grading, subtypes, or their specific radiosensitivity. Another disadvantage of this retrospective study was the lack of a more sophisticated second-level tissue pattern analysis, such as the co-occurrence of anisotropic gradient orientations or calculation of Shannon entropy, ${ }^{12,18}$ which was not available to us, or perfusion data, which we did not acquire during the pretreatment MR examination to assess tumor vascularity. ${ }^{21}$

\section{Conclusions}

The prediction of volumetric response after radiosur- gery can influence therapeutic strategies, such as modifying radiation dose, or strengthen the case for radiosurgery over conventional resection. The results of our texture analysis indicate that DTI-derived parameters have a higher correlation to shrinkage of meningioma volume after GKRS than do data from T1- and T2-weighted sequences. If only routine MR images are available, the SD of T2weighted images can be used to predict treatment success. However, MRI results should be compared to tumor volume reduction and histopathological findings in a prospective study with a larger cohort of patients to strengthen the underlying oncological basis.

\section{Acknowledgments}

We gratefully acknowledge Ms. Cathy Lebron, Ms. Evelyn Borel, Mr. Jairo Santana, Mr. Diego Almeida, Mr. Keithel Brea, and Mr. George Lara for providing valuable contributions to this study.

\section{References}

1. Bauer S, Wiest R, Nolte LP, Reyes M: A survey of MRIbased medical image analysis for brain tumor studies. Phys Med Biol 58:R97-R129, 2013

2. Benjamini Y, Hochberg Y: Controlling the false discovery rate: a practical and powerful approach to multiple testing. J R Stat Soc Series B Stat Methodol 57:289-300, 1995

3. Chaddad A, Desrosiers C, Toews M: Radiomic analysis of multi-contrast brain MRI for the prediction of survival in patients with glioblastoma multiforme. Conf Proc IEEE Eng Med Biol Soc 2016:4035-4038, 2016 
4. Chen TC, Zee CS, Miller CA, Weiss MH, Tang G, Chin L, et al: Magnetic resonance imaging and pathological correlates of meningiomas. Neurosurgery 31:1015-1022, 1992

5. Conturo TE, McKinstry RC, Akbudak E, Robinson BH: Encoding of anisotropic diffusion with tetrahedral gradients: a general mathematical diffusion formalism and experimental results. Magn Reson Med 35:399-412, 1996

6. Earnest F IV, Kelly PJ, Scheithauer BW, Kall BA, Cascino TL, Ehman RL, et al: Cerebral astrocy tomas: histopathologic correlation of MR and CT contrast enhancement with stereotactic biopsy. Radiology 166:823-827, 1988

7. Filippi CG, Edgar MA, Uluğ AM, Prowda JC, Heier LA, Zimmerman RD: Appearance of meningiomas on diffusionweighted images: correlating diffusion constants with histopathologic findings. AJNR Am J Neuroradiol 22:65-72, 2001

8. Georgiadis P, Cavouras D, Kalatzis I, Glotsos D, Athanasiadis E, Kostopoulos S, et al: Enhancing the discrimination accuracy between metastases, gliomas and meningiomas on brain MRI by volumetric textural features and ensemble pattern recognition methods. Magn Reson Imaging 27:120 130, 2009

9. Harrison G, Kano H, Lunsford LD, Flickinger JC, Kondziolka D: Quantitative tumor volumetric responses after Gamma Knife radiosurgery for meningiomas. J Neurosurg 124:146154, 2016

10. Hsu CC, Pai CY, Kao HW, Hsueh CJ, Hsu WL, Lo CP: Do aggressive imaging features correlate with advanced histopathological grade in meningiomas? J Clin Neurosci 17:584-587, 2010

11. Jolapara M, Kesavadas C, Radhakrishnan VV, Thomas B, Gupta AK, Bodhey N, et al: Role of diffusion tensor imaging in differentiating subtypes of meningiomas. J Neuroradiol 37:277-283, 2010

12. Kinoshita M, Sakai M, Arita H, Shofuda T, Chiba Y, Kagawa $\mathrm{N}$, et al: Introduction of high throughput magnetic resonance T2-weighted image texture analysis for WHO grade 2 and 3 gliomas. PLoS One 11:e0164268, 2016

13. Kotrotsou A, Zinn PO, Colen RR: Radiomics in brain tumors: an emerging technique for characterization of tumor environment. Magn Reson Imaging Clin N Am 24:719729, 2016

14. Leemans A, Jeurissen B, Sijbers J, Jones DK: ExploreDTI: a graphical toolbox for processing, analyzing, and visualizing diffusion MR data. Proc Intl Soc Magn Reson Med 17:3537, 2009

15. Li Z, Mao Y, Li H, Yu G, Wan H, Li B: Differentiating brain metastases from different pathological types of lung cancers using texture analysis of T1 postcontrast MR. Magn Reson Med 76:1410-1419, 2016

16. Maiuri F, Iaconetta G, de Divitiis O, Cirillo S, Di Salle F, De Caro ML: Intracranial meningiomas: correlations between MR imaging and histology. Eur J Radiol 31:69-75, 1999

17. Peeken JC, Nüsslin F, Combs SE: "Radio-oncomics": The potential of radiomics in radiation oncology. Strahlenther Onkol 193:767-779, 2017

18. Prasanna P, Tiwari P, Madabhushi A: Co-occurrence of Local Anisotropic Gradient Orientations (CoLlAGe): a new radiomics descriptor. Sci Rep 6:37241, 2016
19. Speckter H, Bido J, Hernandez G, Mejía DR, Suazo L, Valenzuela $S$, et al: Prognostic value of diffusion tensor imaging parameters for Gamma Knife radiosurgery in meningiomas. J Neurosurg 125 (1 Suppl 1):83-88, 2016

20. Suzuki Y, Sugimoto T, Shibuya M, Sugita K, Patel SJ: Meningiomas: correlation between MRI characteristics and operative findings including consistency. Acta Neurochir (Wien) 129:39-46, 1994

21. Toh CH, Wei KC, Chang CN, Peng YW, Ng SH, Wong HF, et al: Assessment of angiographic vascularity of meningiomas with dynamic susceptibility contrast-enhanced perfusionweighted imaging and diffusion tensor imaging. AJNR Am J Neuroradiol 35:263-269, 2014

22. Tropine A, Dellani PD, Glaser M, Bohl J, Plöner T, Vucurevic $\mathrm{G}$, et al: Differentiation of fibroblastic meningiomas from other benign subtypes using diffusion tensor imaging. J Magn Reson Imaging 25:703-708, 2007

23. Vernimmen FJ, Slabbert JP: Assessment of the alpha/ beta ratios for arteriovenous malformations, meningiomas, acoustic neuromas, and the optic chiasma. Int J Radiat Biol 86:486-498, 2010

24. Wang S, Kim S, Zhang Y, Wang L, Lee EB, Syre P, et al: Determination of grade and subtype of meningiomas by using histogram analysis of diffusion-tensor imaging metrics. Radiology 262:584-592, 2012

\section{Disclosures}

The authors report no conflict of interest concerning the materials or methods used in this study or the findings specified in this paper.

\section{Author Contributions}

Conception and design: Speckter, Stoeter. Acquisition of data: all authors. Analysis and interpretation of data: all authors. Drafting the article: Speckter, Stoeter. Critically revising the article: all authors. Reviewed submitted version of manuscript: all authors. Approved the final version of the manuscript on behalf of all authors: Speckter. Statistical analysis: Speckter, Stoeter. Administrative/technical/material support: Speckter, Gonzalez. Study supervision: Speckter.

\section{Supplemental Information \\ Previous Presentations}

This study was presented in part at ALATRO (Asociación IberoLatinoamericana de Terapia Radiante Oncológica) 2017, held in Punta Cana, Dominican Republic, on November 8, 2017, and in full at the 19th Leksell Gamma Knife Society Meeting, held in Dubai, United Arab Emirates, on March 7, 2018.

\section{Correspondence}

Herwin Speckter: Centro Gamma Knife Dominicano, CEDIMAT, Plaza de la Salud, Santo Domingo, Dominican Republic. hspeckter@cedimat.net. 